\title{
The role of serum response factor in hepatocellular carcinoma: Implications for disease progression
}

\author{
CHANG YOUNG KWON ${ }^{1}$, KYUNG RYOUL KIM ${ }^{3}$, HA NA CHOI ${ }^{1}$, MYOUNG JA CHUNG ${ }^{1}$, SANG JAE NOH ${ }^{1}$, \\ DAE GHON KIM ${ }^{2}$, MYOUNG JAE KANG ${ }^{1}$, DONG GEUN LEE ${ }^{1}$ and WOO SUNG MOON ${ }^{1}$
}

\author{
${ }^{1}$ Department of Pathology, ${ }^{2}$ Internal Medicine, Chonbuk National University, Medical School, Institute for Medical Sciences, \\ Research Institute of Clinical Medicine, Jeonju, Chonbuk 561-756; ${ }^{3}$ Forensic Medicine Division, Forensic Medicine \\ Department, National Institute of Scientific Investigation, Seoul 158-707, Korea
}

Received April 27, 2010; Accepted June 23, 2010

DOI: 10.3892/ijo_00000734

\begin{abstract}
Serum response factor (SRF) regulates transcription of the immediate early genes and triggers proliferation, migration and differentiation in several types of cells. We examined the role of SRF in HCC by transfecting the SRF cDNA in HLE cells and the SRF anti-sense cDNA in sarcomatoid HCC cells. The overexpression of SRF in the HLE cells significantly increased the cell growth and proliferation. Overexpression of SRF increased actin polymerization of the HCC cells and induced morphologic changes. The mesenchymal markers vimentin, N-cadherin and RhoA were highly expressed in the SRF-transfected HLE cells. Furthermore, the overexpression of SRF in the HLE cells increased the expression levels of the active form of the B-catenin and Wnt/ ß-catenin target genes, such as c-myc and cyclin D1. The overexpression of SRF significantly enhanced the cell migration and invasiveness of HCC cells. Conversely, inhibition of the SRF expression in the sarcomatoid SH-J1 cells by the SRF anti-sense cDNA significantly decreased migration and invasion through the attenuated expression of mesenchymal markers and the proteins involved in the $\mathrm{Wnt} / \mathrm{B}$-catenin pathway. These results indicate that the overexpression of SRF in HCC cells modulates the Wnt/ß-catenin pathway, and this plays an important role in HCC progression.
\end{abstract}

\section{Introduction}

Hepatocellular carcinoma (HCC) is the sixth most common malignant tumor worldwide with an incidence of 626,000 cases and 598,000 deaths annually, making it the third most common cause of cancer deaths throughout the world (1).

Correspondence to: Dr Woo Sung Moon, Department of Pathology, Chonbuk National University, Medical School, Jeonju, Korea

E-mail:mws@chonbuk.ac.kr

Key words: carcinoma, hepatocellular, serum response factor, ß-catenin, metastasis
Considerable progress has been made over the past few decades in diagnosing and treatment of HCC. However, HCC is still associated with a high rate of mortality, and the prognosis of this tumor is poor even with treatment that is considered potentially curative (2). The molecular changes and mechanisms that regulate the development and progression of HCC remain unclear.

Serum response factor (SRF) is a transcription factor of the MADS box family (3-6). To date, the DNA binding sites for SRF, called the serum response elements (SREs), have been found in the promoters of approximately 300 different genes (3-8). Studies have also shown that SRF is involved in various cellular processes such as the expressions of immediate early genes like c-fos and Egr-1 and tissuespecific genes, cell proliferation, differentiation and apoptosis (8-13). While the function of SRF has been intensely studied in muscle and the nervous system, little is known about the contribution of SRF to hepatic carcinogenesis and its functional role. A recent study has demonstrated that SRF plays a role in tumor progression and specifically in the mesenchymal transition of epithelial tumor cells (14). More recently, Park et al have also reported that the expression of SRF is correlated with tumor cell de-differentiation and SRF is involved in epithelial-mesenchymal transition (EMT) in HCC cells (15).

The role of the Wnt/ß-catenin signaling pathway in liver biology has come to the forefront over the last several years. Advances have been made in understanding the role of Bcatenin in the development of various liver diseases (16). Studies of pathologic specimens and rodent models of liver diseases have demonstrated aberrations in the $\mathrm{Wnt} / \mathrm{B}$-catenin signaling pathway in pathological conditions ranging from hepatitis to HCC (16). B-catenin is the chief downstream effector of the canonical Wnt signaling pathway. In a normal steady state where B-catenin activation is not necessary or in the absence of a Wnt signal, the free monomeric form of B-catenin in the cytoplasm is actively targeted for degradation by ubiquitination $(17,18)$. In the presence of a Wnt signal, the degradation of $\beta$-catenin is inhibited and the consequence is the stabilization of cytoplasmic B-catenin protein, which translocates to the nucleus and consequently induces the expression of Wnt target genes, including c-myc and cyclin D1. 
Dysfunctional $\beta$-catenin signaling, which creates continuous transcription of many target genes supporting cell proliferation, has now been documented in a wide range of cancers, including HCC. Numerous studies suggest that the activation of the Wnt/ß-catenin signaling pathway plays an important role in human tumorigenesis $(16,19-21)$. However, the functional role(s) of SRF and its relationship to the Bcatenin signaling pathway in HCC are not clear.

In the present study, we examined a) the effect of SRF in HCC cell growth and proliferation, b) SRF and its relationship to EMT and the change of the expression levels of EMTrelated proteins in $\mathrm{HCCs}, \mathrm{c}$ ) the effect of SRF in the B-catenin pathway and d) the role of SRF in the migration and invasion of HCC cells.

\section{Materials and methods}

HCC cell lines. The human HCC cell line HLE was purchased from the Health Science Research Resources Bank (Osaka, Japan). In addition, we used the sarcomatoid HCC cell line, designated as SH-J1, which was established in our laboratory (22). The HCC cells were maintained in Dulbecco's modified Eagle's medium (DMEM) supplemented with penicillin and streptomycin $(100 \mathrm{U} / \mathrm{ml})$ and $10 \%$ fetal bovine serum (Gibco BRL, Gaithersburg, MD, USA), and the cells were grown at $37^{\circ} \mathrm{C}$ in a humidified $5 \% \mathrm{CO}_{2}$ incubator.

Stable transfection of SRF cDNA and anti-sense SRF cDNA. The expression Vector pcDNA3.1-full-length SRF cDNA (accession no. J03161) or an anti-sense SRF was kindly provided by J. Chai, Department of Medicine, University of California, CA, USA (23). The human HLE and SH-J1 cells were cultured in DMEM supplemented with $10 \%$ FBS and antibiotics to $90 \%$ confluence of growth. Gene transfection was performed according to the protocol of the lipofectin manufacturer (Invitrogen, Carlsbad, CA, USA). Briefly, the cells were rinsed with serum-free medium without antibiotics, and the cells were transfected separately with pcDNA 3.1-SRF or anti-sense SRF $1 \mu \mathrm{g} /$ lipofectin 8-10 $\mu \mathrm{l}$, or pcDNA3.1 $1 \mu \mathrm{g} /$ lipofectin 8-10 $\mu \mathrm{l}$ (mock control), followed by incubation at $37^{\circ} \mathrm{C}$ in a $5 \% \mathrm{CO}_{2}$ incubator for $12 \mathrm{~h}$. The medium was then replaced by DMEM with $10 \%$ FBS without antibiotics. Eighteen hours later, the cells in each group were taken out to detect the transient expression of SRF or antisense SRF by Western blot methods, whereas the other cells were continuously cultured for the stable expression of SRF or anti-sense SRF. The cells were subcultured in DMEM with $10 \%$ FBS with G418 $(600-800 \mu \mathrm{g} / \mathrm{ml})$. After 2 weeks of culture in the presence of G418, seven and six positive clones were respectively selected and transferred into 24-well dishes. The selected clones were taken for identification and then frozen until further study.

Western blotting. The total protein was extracted in $1 \%$ Nonidet P-40 buffer (50 mM Tris-HCl, $150 \mathrm{mM} \mathrm{NaCl}, 1 \mathrm{mM}$ EDTA) that was supplemented with a protease inhibitor cocktail (Sigma, St. Louis, MO, USA) and phosphatase inhibitor cocktail I and II (Sigma), and the protein was incubated on ice for $10 \mathrm{~min}$ and centrifuged at $12,000 \mathrm{x} \mathrm{g}$ for $10 \mathrm{~min}$ at $4^{\circ} \mathrm{C}$. The protein concentration was determined by using the
Coomassie blue method (Bio-Rad protein assay, Bio-Rad, Richmond, CA, USA). The protein samples were separated by electrophoresis on an $8 \%$ SDS-polyacrylamide gel, and the separated proteins were electrotransferred to a polyvinylidene difluoride (PVDF) membrane using a semidry transfer method (Bio-Rad). The membrane was then blocked with $5 \%$ non-fat dry milk in Tris-buffered saline (TBS)- $0.1 \%$ Tween-20 (15 mM NaCl, 100 mM Tris-HCl, pH 7.5) for $1 \mathrm{~h}$ to reduce the non-specific binding. The membrane was incubated with anti-SRF, anti-vimentin, anti-c-fos, anti-Egr-1 (Santa Cruz Biotechnology, Santa Cruz, CA, USA) anti-Ecadherin, anti-N-cadherin, anti- $\beta$-catenin (BD Biosciences, San Jose, CA, USA), anti-dephospho- $\beta$-catenin (Upstate, Lake Placid, NY, USA), anti-c-myc, anti-cyclin D1 and antiRhoA (Santa Cruz Biotechnology) overnight at $4^{\circ} \mathrm{C}$. After washing 3 times, the membrane was incubated for $1 \mathrm{~h}$ at room temperature with the corresponding secondary antibodies, and the immune complexes were visualized using an ECL detection system (Amersham Biosciences, Buckinghamshire, UK); they were then exposed to a luminescent image analyzer (LAS-3000, Fuji Film, Tokyo, Japan). Equal loading of proteins in each lane was confirmed by probing the membrane with mouse anti- $\beta$-actin (Sigma).

Cell growth and proliferation assay. The effects of the SRF gene on the mitochondria activity and DNA synthesis of cells were assessed by WST-1 and BrdU (Bromodeoxyuridine) incorporation assay (Roche Applied Science, Mannheim, Germany). The HLE and SH-J1 cells were grown on a 96-well culture plate $\left(3-5 \times 10^{3}\right.$ per well). After $24-36 \mathrm{~h}$, the cells were serum-starved for 12-16 h and then stimulated with $20 \%$ FBS for $8 \mathrm{~h}$. The incorporated BrdU and WST-1 were measured colorimetrically using a microtiter plate reader (Bio-Rad) at $450 \mathrm{~nm}$.

Staining for $G$-actin and $F$-actin. The HLE cells were seeded on glass cover slides on 24-well culture plates. After 24-36 h, the cells were serum-starved for 12-16 $\mathrm{h}$ and then stimulated with $20 \%$ FBS for $8 \mathrm{~h}$. The cells were fixed with $3.7 \%$ formaldehyde and permeabilized with $0.1 \%$ Triton X-100 in PBS. The cells were stained with Alexa Fluor 594-conjugated deoxyribonuclease $\pi$ (DNase I) and Alexa Fluor 495-conjugated phalloidin (Molecular Probes, Eugene, OR, USA), according to the manufacturer's protocol. To visualize the nucleus, the cells were incubated with DAPI (Molecular Probes) for $3 \mathrm{~min}$. The cells were observed with the use of a fluorescence microscope.

In vitro migration assay. The HLE and $\mathrm{SH}-\mathrm{J} 1$ cell migration assays were performed using a 24-transwell migration chamber (Corning Life Sciences, Acton, MA, USA). The upper and lower chambers were separated by a polyvinyl-pyrrolidonefree polycarbonate membrane with an $8-\mu \mathrm{m}$ pore size. Cells $\left(4 \times 10^{4}\right.$ per well) were suspended in serum-free medium and placed in the upper chamber. Medium containing 2\% FBS was placed as the chemo-attractant source. Ten hours later, the cells on the upper surface of the filter were wiped with a cotton swab. The cells on the lower surface of the filters were fixed and stained with Diff-Quik solution (Dade Behring, Newark, NJ, USA). The cells that migrated to the lower 

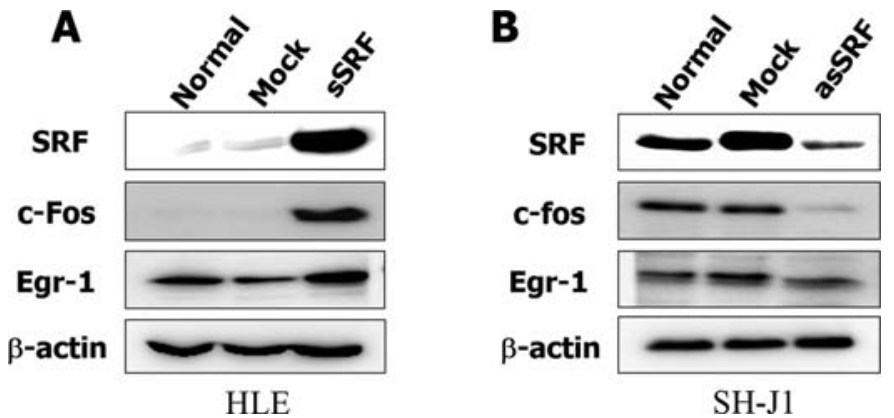

Figure 1. (A) The enhanced expression of SRF and the immediate early genes in HLE cells transfected with the SRF gene. (B) The decreased expression of SRF and the immediate early genes in SH-J1 cells transfected with the anti-SRF gene.

\section{A}

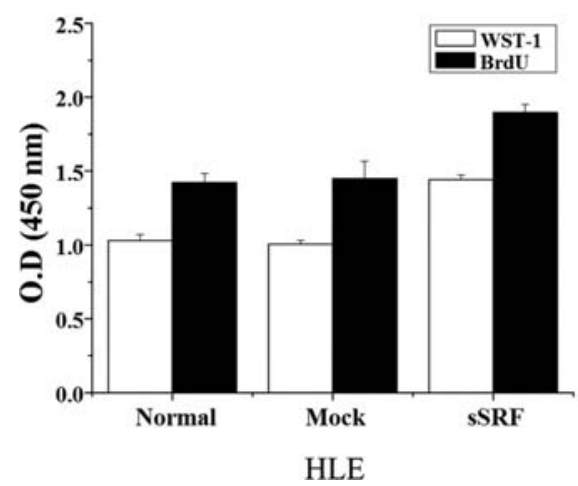

B

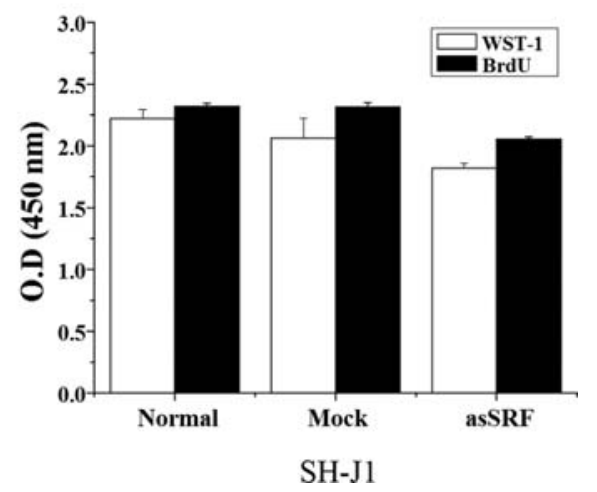

Figure 2. (A) Transfection of the SRF gene significantly enhanced the cell growth and proliferation of HLE cells. (B) In contrast, the cell growth and proliferation of sarcomatoid SH-J1 cells were decreased when the SRF expression was blocked by anti-sense SRF cDNA.

surface of the filter were counted under a light microscope at x200 magnification in ten randomly selected fields per well.

In vitro invasion assay. The HLE and $\mathrm{SH}-\mathrm{J} 1$ cell invasion assays were performed using a 24-transwell BioCoat Matrigel invasion chamber (BD Biosciences) with an $8-\mu \mathrm{m}$ pore size polyvinyl-pyrrolidone-free polycarbonate membrane. The cells $\left(6 \times 10^{4}\right.$ per well) were suspended in serum-free medium and placed in the upper chamber. FBS (5\%) containing medium was placed as the chemo-attractant source. Twentytwo hours later, the cells on the upper surface of the filter were wiped with a cotton swab. The cells on the lower surface of the filters were fixed and stained with Diff-Quik solution (Dade Behring). The cells that invaded to the lower surface of the filter were counted under a light microscope at x200 magnification in ten randomly selected fields per well.

Statistical analysis. The data are presented as the mean \pm SD. Student's t-test was used to determine statistical significance. p-values $<0.05$ were considered statistically significant.

\section{Results}

Transfection of SRF cDNA promotes the expression of immediate early genes. First, we analyzed the expression levels of SRF itself and the SRF target genes, $c$-Fos and Egr-1, in the SRF-transfected HCC cells by Western blotting. In the
$S R F$-transfected HLE cells, SRF, c-Fos and Egr-1 were increased by 20-, 30- and 3-fold, respectively (Fig. 1A). Conversely, inhibition of the SRF expression in the SH-J1 cells significantly decreased the expression of c-Fos and Egr-1 (Fig. 1B).

Effects of the SRF expression on cell growth and cell proliferation. The SRF-overexpressed HLE cells showed significantly increased cell growth and proliferation by 44 and $30 \%$ when compared to those of the control, respectively $(\mathrm{p}<0.001)$ (Fig. 2A). In contrast, the cell growth and proliferation of the sarcomatoid SH-J1 cells were decreased when the SRF expression was blocked by anti-sense SRF cDNA (Fig. 2B).

Morphologic changes and increased actin polymerization in the SRF-overexpressed HCC cells. Next, we examined the effect of SRF on actin polymerization in the SRF cDNAtransfected HLE cells. DNase I is an endonuclease that has a high binding affinity for the subdomain 2 (the pointed end) of G-actin. It binds tightly to $\mathrm{G}$-actin in a 1:1 ratio. Phalloidin is a peptide isolated from the mushroom Amanita phalloides. It specifically binds to the junctions between the actin subunits in F-actin. Overexpression of SRF in the HLE cells increased actin polymerization and the cells showed more extensive lamellipodia, compared with that of the control (Fig. 3A). Furthermore, SRF overexpressing cells, but not the 
A
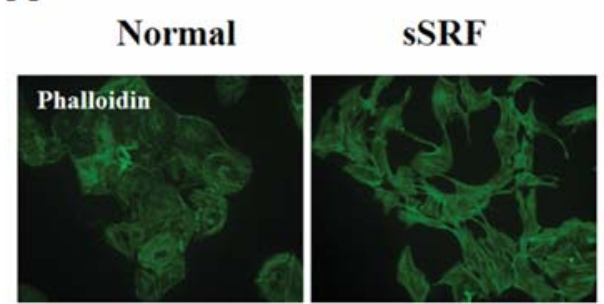

B
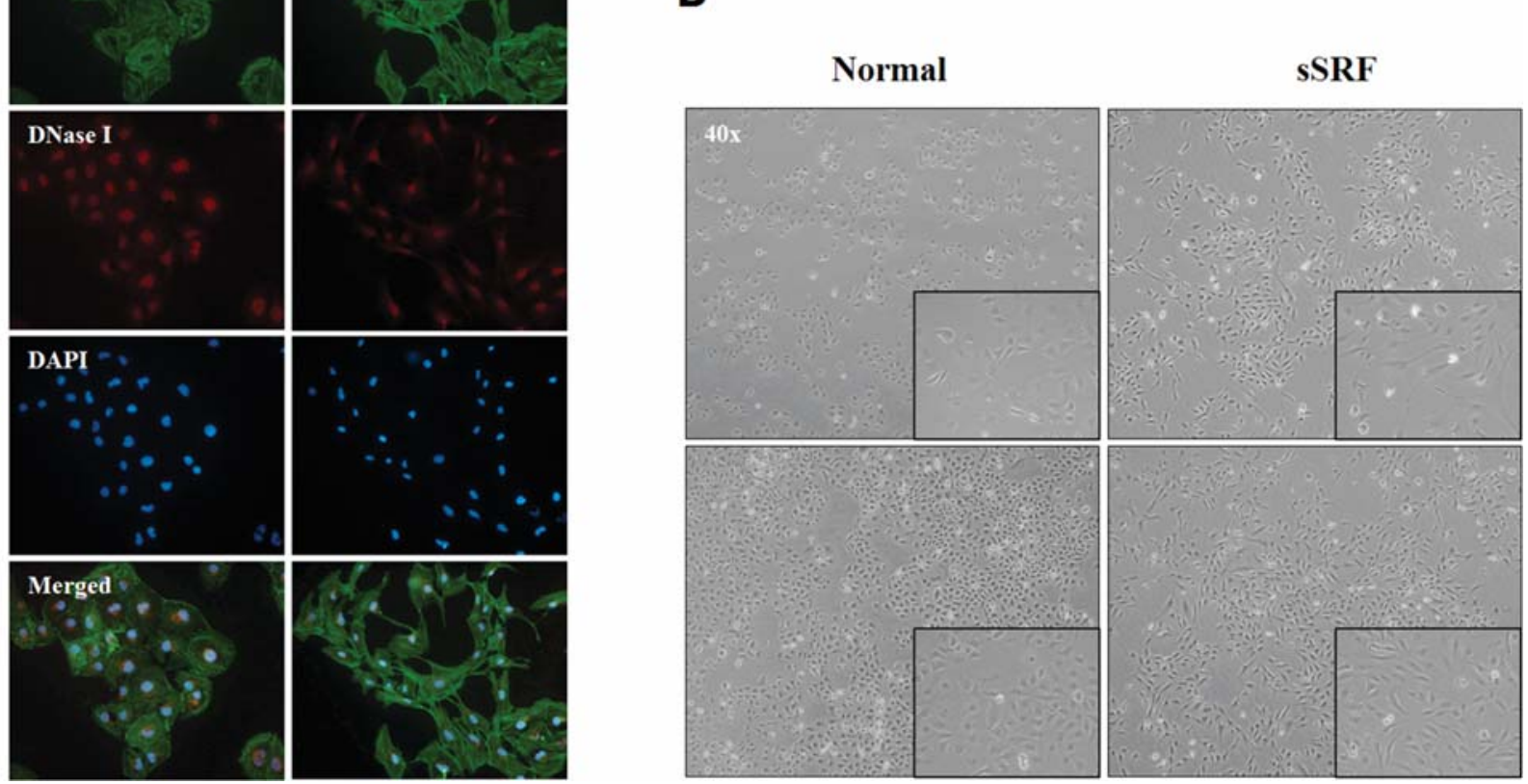

Figure 3. (A) The overexpression of SRF in the HLE cells increased actin polymerization and the cells showed more extensive lamellipodia, compared with the control. (B) The overexpression of SRF induced a spindle-like, fibroblast morphology that is typical of mesenchymal transition.
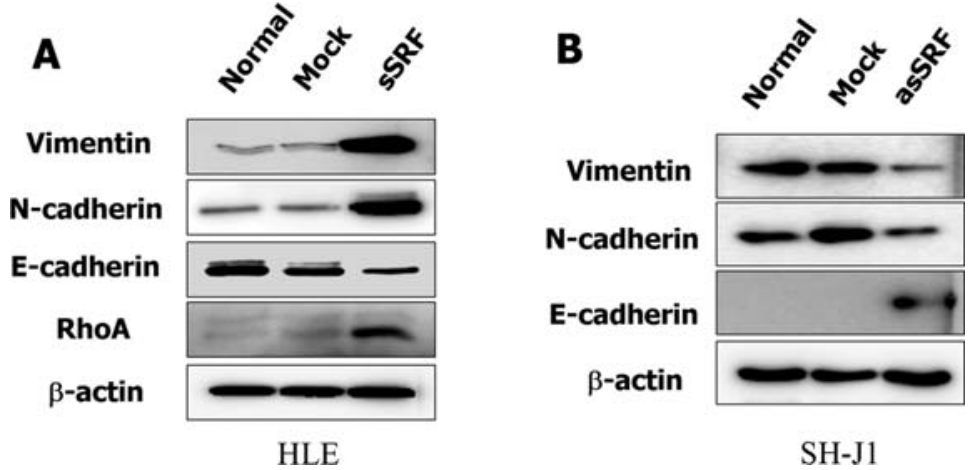

Figure 4. (A) The enhanced expression of mesenchymal markers vimentin, N-cadherin and RhoA in the HLE cells transfected with the SRF gene. The overexpression of SRF resulted in a decrease in the expression level of E-cadherin as compared to the expression level determined in the control cells. (B) Conversely, the inhibition of the SRF expression in the sarcomatoid SH-J1 cells by anti-sense SRF cDNA increased the expression of E-cadherin.

control cells, assumed a spindle-like, fibroblast morphology that is typical of mesenchymal transition (Fig. 3B).

Overexpression of SRF induced epithelial-mesenchymal transition and activated the $\beta$-catenin pathway. The representative mesenchymal markers vimentin, $\mathrm{N}$-cadherin and RhoA were highly increased by 50-, 3.3- and 10-fold, respectively, in the $S R F$-transfected HLE cell line (Fig. 4A). Furthermore, overexpression of SRF in the HCC cells decreased the expression level of E-cadherin, which is a representative epithelial adhesion molecule. Conversely, the inhibition of the SRF expression in the sarcomatoid SH-J1 cells by anti-sense SRF cDNA decreased the expression of $\mathrm{N}$-cadherin with simul- taneous recovery of the E-cadherin expression (Fig. 4B). At the same time, the overexpression of SRF increased the expression levels of the active form of $\beta$-catenin and the Wnt/ B-catenin target genes, such as c-myc and cyclin D1. In the $S R F$-transfected HLE cells, B-catenin, activated nuclear ß-catenin, c-myc and cyclin D1 were increased by 1.4-, 3-, 3.5- and 5-fold, respectively (Fig. 5A). Conversely, inhibition of the SRF expression in the sarcomatoid SH-J1 cells by antisense SRF cDNA decreased the expression of activated ß-catenin, c-myc and cyclin D1 (Fig. 5B).

Overexpression of SRF accelerates cell migration and invasion. The overexpression of SRF in the HLE cells significantly 


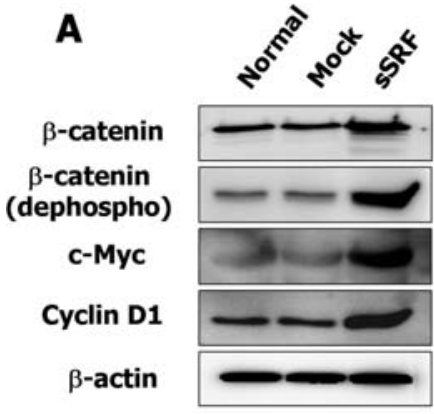

HLE

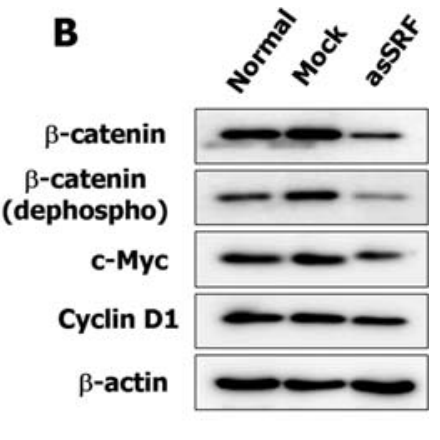

SH-J1

Figure 5. (A) The enhanced expression levels of the active form of the B-catenin and Wnt/B-catenin target genes, such as c-myc and cyclin D1. (B) Conversely, the inhibition of the SRF expression in the sarcomatoid SH-J1 cells by anti-sense SRF cDNA decreased the expression of activated B-catenin, c-myc and cyclin D1.

A
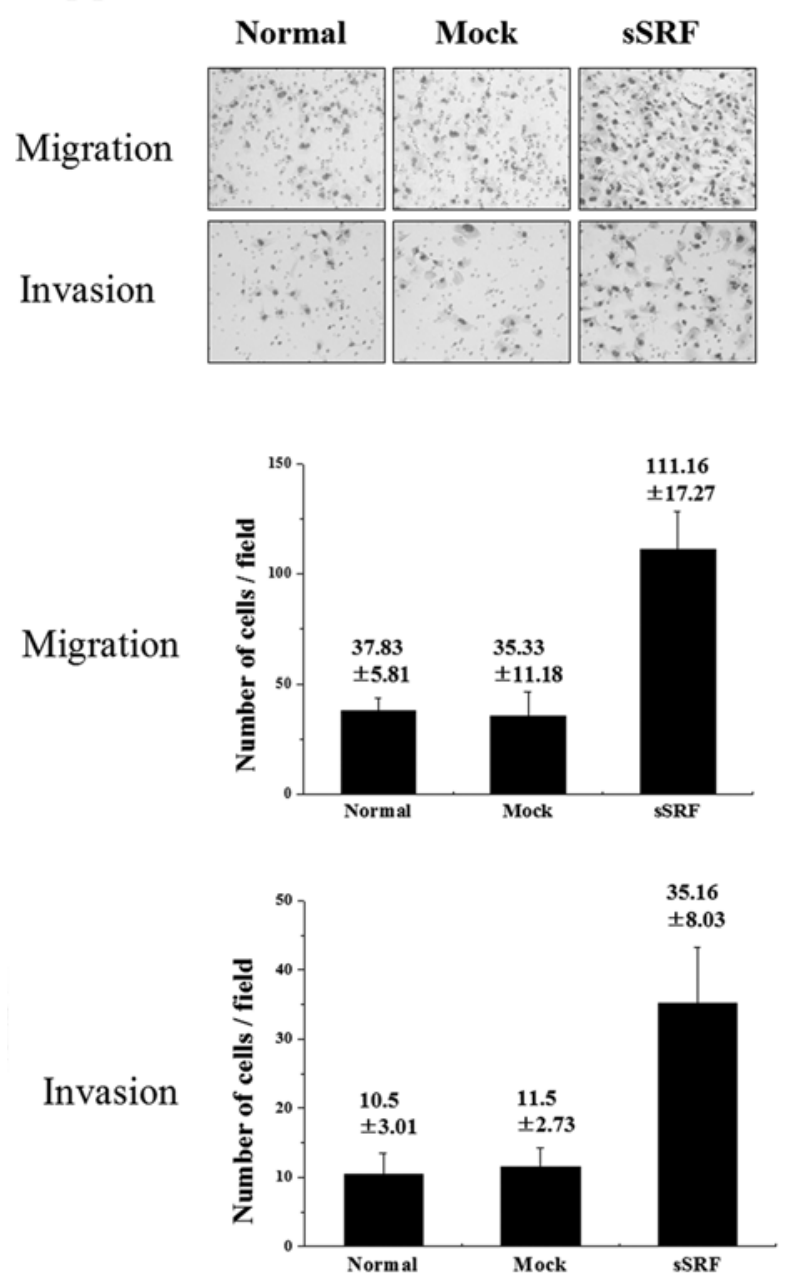

HLE
B

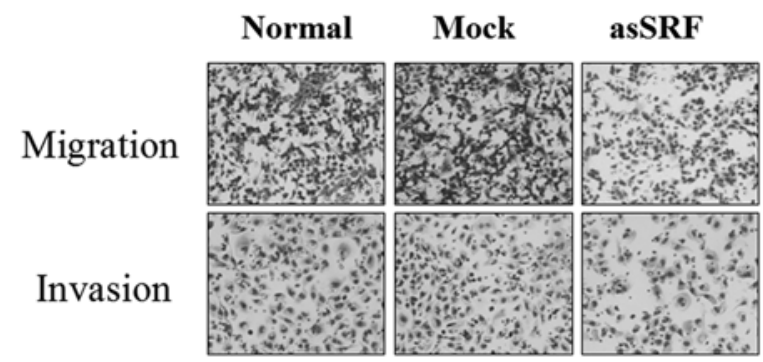

Migration

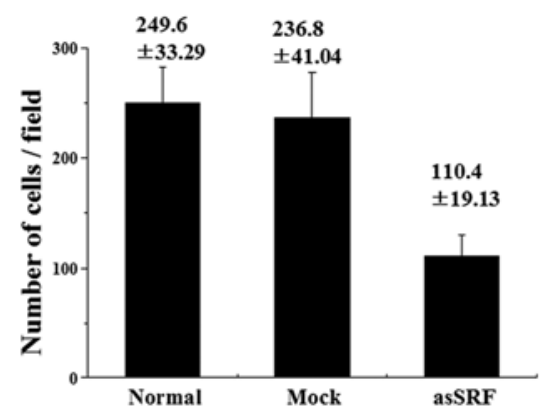

Invasion

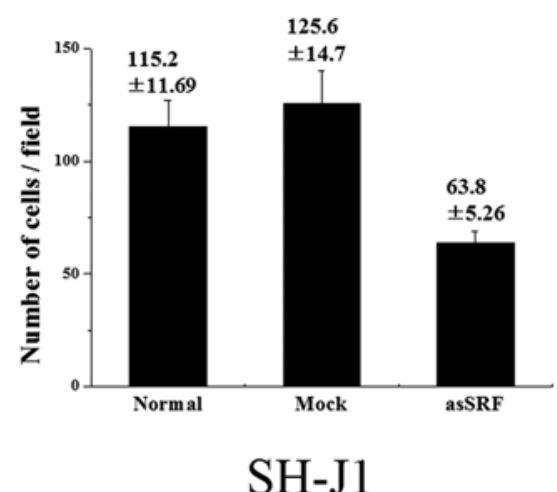

Figure 6. In vitro cell migration and invasion assay. (A) For the SRF-transfected HLE cells, cell migration was increased by 3.3-fold when compared with that of the control. The SRF-transfected HLE cell invasion was increased 3-fold when compared with that of the control. (B) For the anti-sense SRF transfected SHJ-1 cells, cell migration and invasion were decreased 2.1- and 1.9-fold, respectively, when compared with those of the control. The experiment was independently repeated three times $(\mathrm{p}<0.05)$.

increased cell migration and invasion 3.3- and 3-fold when compared with those of the control, respectively $(\mathrm{p}<0.05)$. Conversely, inhibition of the SRF expression in the SH-J1 cells significantly decreased cell migration and invasion 2.1and 1.9-fold when compared with those of the control, respectively $(\mathrm{p}<0.05)$ (Fig. 6A and B). 


\section{Discussion}

This study demonstrated the following for the first time: a) the overexpression of SRF significantly increased cell growth and proliferation in HLE cells, b) the overexpression of SRF induced morphologic changes and increased actin polymerization in the HLE cells, c) mesenchymal markers were highly expressed in the SRF-tansfected HLE cells, d) the overexpression of SRF increased the expression levels of the active form of $\beta$-catenin and the $\mathrm{Wnt} / \mathrm{B}$-catenin target genes, such as c-myc and cyclin D1, and e) the overexpression of SRF enhanced the cell motility and invasiveness of HCC cells. Furthermore, inhibition of the SRF expression in the sarcomatoid SH-J1 cells by anti-sense SRF cDNA significantly decreased cell migration and invasion through attenuating the expression of mesenchymal markers and proteins involved in the $\mathrm{Wnt} / \mathrm{B}$-catenin pathway. These findings clearly indicate the important role of SRF in HCC progression, through not only EMT, but also through modification of the Wnt/ß-catenin pathway of the carcinoma cells.

In this study, we found that the overexpression of SRF significantly increased cell growth and proliferation in the HLE cells. Furthermore, the cell growth and proliferation of the sarcomatoid SH-J1 cells were decreased when the SRF expression was blocked by anti-sense SRF cDNA. It is well known that activation of the immediate early genes in quiescent cells following addition of serum is important for cells to exit G0 and re-enter the G1 phase of the cell cycle. Similar to our results, when anti-SRF antibody was introduced to the quiescent rat embryonic fibroblasts, it abolished the serum-stimulated c-fos induction and suppressed DNA synthesis $(24,25)$. SRF was also found to act downstream of phosphoinositide 3-kinase signaling and it was required for phosphoinositide 3-kinase-induced cell proliferation (26). More recently, Sun et al have reported that SRF is essential for maintenance of hepatocyte viability and proliferation (27). Taken together, our data suggest that the SRF expression appears to be necessary for the cell growth and cell cycle progression of HCC cells.

Epithelial mesenchymal transition (EMT) is a switch of polarized epithelial cells to a migratory fibroblastoid phenotype, and EMT is increasingly considered to be an important event during malignant epithelial tumor progression and metastasis $(28,29)$. The hallmark of classic EMT is the change from the epithelial phenotype to the expression of mesenchymal markers and this is accompanied by a loss of tight cell-cell adhesion and the acquisition of the spindle cell morphology (28-35). In this study, the representative mesenchymal markers vimentin, N-cadherin and RhoA were significantly up-regulated, while a representative epithelial adhesion molecule E-cadherin was down-regulated in the SRF-transfected HLE cell line. We also found that the overexpression of SRF induces spindle-shape, fibroblast morphologic change of the HCC cells along with extensive lamellipodia formation. Conversely, inhibition of the SRF expression in the sarcomatoid SH-J1 cells by anti-sense SRF cDNA increased the expression of E-cadherin, indicating the cells had acquired the epithelial phenotype. A previous study has demonstrated that the human epithelial HCC cell lines HLE, Huh-7 and HepG2 do not express vimentin and they showed a minimal expression of SRF. However, the sarcomatoid HCC cell line, designated as SH-J1, highly expressed vimentin and SRF. Furthermore, the expression of SRF in HCC cancer cells was previously shown to be significantly associated with tumor de-differentiation in resected HCC specimens (15). Together with the fact that carcinomas showing EMT are mostly high-grade carcinomas (33-35), our findings suggest that an aberrant SRF expression in HCC cancer cells leads to tumor progression through EMT. In agreement with our findings, a previous study has demonstrated that the expression of SRF is up-regulated and highly active in tumor cells that have undergone the EMT and that have acquired a mesenchymal phenotype (14). Furthermore, the expression of SRF was up-regulated and it enhanced the expression of the SRF target gene $c-F o s$, and this was associated with tumor progression and metastasis in papillary carcinoma of the thyroid (36).

Wnt/ß-catenin signaling is known for its role in embryogenesis as well as carcinogenesis. In the liver, Wnt/B-catenin signaling plays many critical roles during hepatic development and regeneration, and its dysregulation is evident in the aberrant hepatic growth of various liver tumors (16-18,20,37$40)$. Wnt/ß-catenin activation has also been implicated in many other cancers and it is one of the important aberrant pathways identified in the HCC in animals and humans $(17,18,37-40)$. In this study, we found that the overexpression of SRF in HCC cells decreased the expression of E-cadherin and increased the nuclear (dephospho)- $\beta$-catenin expression with subsequent up-regulation of the c-myc and cyclin D1 expressions. We further demonstrated that inhibition of the SRF expression in sarcomatoid SH-J1 cells by anti-sense SRF cDNA decreased the expression of activated B-catenin, c-myc and cyclin D1. The association between SRF overexpression and the increased nuclear translocation of $ß$-catenin found in our study may be of importance as B-catenin signaling is strongly linked to hepatocellular carcinogenesis (38-40). The accumulation of $\beta$-catenin in the nucleus induced by SRF may promote an association with $\mathrm{TCF} / \mathrm{LEF}$ transcription factors and affect the transcription of proteins such as cyclin D1 and c-myc, which are regulated by B-catenin (41-43). Although it is not clear whether the overexpression of SRF itself or the regulation of one or more of its target genes is responsible for the activation of $\beta$-catenin, the above findings indicate a critical role of SRF in modulating the $\mathrm{Wnt} / \mathrm{B}$-catenin pathway in HCC.

The morbidity and mortality of patients with cancer is predominantly the result of tumor invasion and metastasis. In our present study, the overexpression of SRF induced increased actin polymerization in HCC cells and enhanced cell motility and invasiveness in vitro. Cell migration requires reorganization of cytoskeletal structures, and particularly polymerization of G-actin to F-actin and the assembly of F-actin into stress fibers. Serum response factor is a master regulator of the actin cytoskeleton and contractile apparatus (44). Our data are consistent with a previous study showing that the overexpression of SRF in RGM1 and A7R5 cells accelerates the migration and proliferation of these cells by promoting actin polymerization during healing of gastric ulcer (23). Moreover, the forced overexpression of SRF in an esophageal epithelial cell line (Het1A) and a rat connective 
tissue fibroblast cell line (Rat1-R12) induced smooth muscle $\alpha$-actin and extensive actin stress fiber formation in both cell lines, suggesting that upregulation of SRF in either epithelial cells or fibroblasts can transform these cells into myofibroblasts (45). Besides actin, SRF also controls transcriptional activation of other cytoskeletal genes such as myosin, vinculin, zyxin, and talin (8), the products of which are also important for cell migration. Our study does not exclude the possibility that SRF overexpression in the HCC cells might have also increased these cytoskeletal components.

In conclusion, our study indicates that the expression of SRF is closely related to EMT and the growth of HCC. Furthermore, SRF appears to activate the B-catenin pathway and to promote migration and invasion in human HCC cells.

\section{Acknowledgments}

This work was supported by the National Research Foundation of Korea (NRF) grant funded by the Korea government (MEST) (2009-0072205) and by the Ministry of Science and Technology (MoST)/Korea Science \& Engineering Foundation (KOSEF) through the Diabetes Research Center at Chonbuk National University (R13-2008-005-0000-0).

\section{References}

1. Parkin DM, Bray F, Ferlay J and Pisani P: Global cancer statistics, 2002. CA Cancer J Clin 55: 74-108, 2005.

2. Befeler AS and Di Bisceglie AM: Hepatocellular carcinoma: diagnosis and treatment. Gastroenterology 122: 1609-1619 2002.

3. Treisman R: Identification of a protein-binding site that mediates transcriptional response of the $\mathrm{c}$-fos gene to serum factors. Cell 46: 567-574, 1986.

4. Treisman R: Identification and purification of a polypeptide that binds to the c-fos serum response element. EMBO J 6: 2711-2717, 1987.

5. Shore $P$ and Sharrocks AD: The MADS-box family of transcription factors. Eur J Biochem 229: 1-3, 1995

6. Norman C, Runswick M, Pollock R and Treisman R: Isolation and properties of cDNA clones encoding SRF, a transcription factor that binds to the c-fos serum response element. Cell 55: 989-1003, 1988.

7. Miano JM: Serum response factor: toggling between disparate programs of gene expression. J Mol Cell Cardiol 35: 577-593, 2003.

8. Chai J and Tarnawski AS: Serum response factor: discovery, biochemistry, biological roles and implications for tissue injury healing. J Physiol Pharmacol 53: 147-157, 2002.

9. Schratt G, Weinhold B, Lundberg AS, Schuck S, Berger J, Schwarz H, Weinberg RA, Ruther U and Nordheim A: Serum response factor is required for immediate-early gene activation yet is dispensable for proliferation of embryonic stem cells. Mol Cell Biol 21: 2933-2943, 2001.

10. Camoretti-Mercado B, Liu HW, Halayko AJ, Forsythe SM, Kyle JW, Li B, Fu Y, McConville J, Kogut P, Vieira JE, Patel NM, Hershenson MB, Fuchs E, Sinha S, Miano JM, Parmacek MS, Burkhardt JK and Solway J: Physiological control of smooth muscle-specific gene expression through regulated nuclear translocation of serum response factor. J Biol Chem 275: 30387-30393, 2000

11. Ding W, Gao S and Scott RE: Senescence represses the nuclear localization of the serum response factor and differentiation regulates its nuclear localization with lineage specificity. J Cell Sci 114: 1011-1018, 2001.

12. Bertolotto C, Ricci JE, Luciano F, Mari B, Chambard JC and Auberger P: Cleavage of the serum response factor during death receptor-induced apoptosis results in an inhibition of the c-FOS promoter transcriptional activity. J Biol Chem 275: 12941-12947, 2000 .
13. Zhang X, Chai J, Azhar G, Sheridan P, Borras AM, Furr MC, Khrapko K, Lawitts J, Misra RP and Wei JY: Early postnatal cardiac changes and premature death in transgenic mice overexpressing a mutant form of serum response factor. J Biol Chem 276: 40033-40040, 2001.

14. Psichari E, Balmain A, Plows D, Zoumpourlis V and Pintzas A: High activity of serum response factor in the mesenchymal transition of epithelial tumor cells is regulated by RhoA signaling. J Biol Chem 277: 29490-29495, 2002.

15. Park MY, Kim KR, Park HS, Park BH, Choi HN, Jang KY, Chung MJ, Kang MJ, Lee DG and Moon WS: Expression of the serum response factor in hepatocellular carcinoma: Implications for epithelial-mesenchymal transition. Int J Oncol 31: 1309-1315, 2007.

16. Thompson MD and Monga SP: WNT/ß-catenin signaling in liver health and disease. Hepatology 45: 1298-1305, 2007.

17. Brantjes H, Barker N, van Es J and Clevers H. TCF: Lady Justice casting the final verdict on the outcome of Wnt signaling. Biol Chem 383: 255-261, 2002.

18. Nelson WJ and Nusse R: Convergence of Wnt, beta-catenin, and cadherin pathways. Science 303: 1483-1487, 2004.

19. Paul S and Dey A: Wnt signaling and cancer development: therapeutic implication. Neoplasma 55: 165-176, 2008.

20. Nejak-Bowen KN, Thompson MD, Singh S, Bowen WC Jr, Dar MJ, Khillan J, Dai C and Monga SP: Accelerated liver regeneration and hepatocarcinogenesis in mice overexpressing serine-45 mutant beta-catenin. Hepatology 51: 1603-1613, 2010.

21. Schneikert J and Behrens J: The canonical Wnt signalling pathway and its APC partner in colon cancer development. Gut 56: 417-425, 2007.

22. Kim DG, Park SY, Kim H, Chun YH, Moon WS and Park SH: A comprehensive karyotypic analysis on a newly established sarcomatoid hepatocellular carcinoma cell line SH-J1 by comparative genomic hybridization and chromosome painting. Cancer Genet Cytogenet 132: 120-124, 2002.

23. Chai J, Baatar D and Tarnawski A: Serum response factor promotes re-epithelialization and muscular structure restoration during gastric ulcer healing. Gastroenterology 126: 1809-1818, 2004.

24. Kovary $\mathrm{K}$ and Bravo R: The jun and fos protein families are both required for cell cycle progression in fibroblasts. Mol Cell Biol 11: 4466-4472, 1991.

25. Riabowol KT, Vosatka RJ, Ziff EB, Lamb NJ and Feramisco JR: Microinjection of fos-specific antibodies blocks DNA synthesis in fibroblast cells. Mol Cell Biol 8: 1670-1676, 1988.

26. Poser S, Impey S, Trinh K, Xia Z and Storm DR: SRFdependent gene expression is required for PI3-kinase-regulated cell proliferation. EMBO J 19: 4955-4966, 2000.

27. Sun K, Battle MA, Misra RP and Duncan SA: Hepatocyte expression of serum response factor is essential for liver function, hepatocyte proliferation and survival, and postnatal body growth in mice. Hepatology 49: 1645-1654, 2009.

28. Boyer B, Valles AM and Edme N: Induction and regulation of epithelial-mesenchymal transitions. Biochem Pharmacol 60: 1091-1099, 2000.

29. Thiery JP: Epithelial-mesenchymal transitions in tumour progression. Nat Rev Cancer 2: 442-454, 2002.

30. Thompson EW and Newgreen DF: Carcinoma invasion and metastasis: a role for epithelial-mesenchymal transition? Cancer Res 65: 5991-5995, 2005.

31. Hay ED and Zuk A: Transformations between epithelium and mesenchyme: normal, pathological, and experimentally induced. Am J Kidney Dis 26: 678-690, 1995.

32. Hu L, Lau SH, Tzang CH, Wen JM, Wang W, Xie D, Huang M, Wang Y, Wu MC, Huang JF, Zeng WF, Sham JS, Yang M and Guan XY: Association of vimentin overexpression and hepatocellular carcinoma metastasis. Oncogene 23: 298-302, 2004.

33. Hendrix MJ, Seftor EA, Seftor RE and Trevor KT: Experimental co-expression of vimentin and keratin intermediate filaments in human breast cancer cells results in phenotypic interconversion and increased invasive behavior. Am J Pathol 150: 483-495, 1997.

34. Gilles C, Polette M, Piette J, Delvigne AC, Thompson EW, Foidart JM and Birembaut P: Vimentin expression in cervical carcinomas: association with invasive and migratory potential. J Pathol 180: 175-180, 1996.

35. Lang SH, Hyde C, Reid IN, Hitchcock IS, Hart CA, Bryden AA, Villette JM, Stower MJ and Maitland NJ: Enhanced expression of vimentin in motile prostate cell lines and in poorly differentiated and metastatic prostate carcinoma. Prostate 52: 253-263, 2002. 
36. Kim HJ, Kim KR, Park HS, Jang KY, Chung MJ, Shong M and Moon WS: The expression and role of serum response factor in papillary carcinoma of the thyroid. Int J Oncol 35: 49-55, 2009.

37. Wong CM, Fan ST and Ng IO: Beta-catenin mutation and overexpression in hepatocellular carcinoma: clinicopathologic and prognostic significance. Cancer 92: 136-145, 2001.

38. Laurent-Puig P, Legoix P, Bluteau O, Belghiti J, Franco D, Binot F, Monges G, Thomas G, Bioulac-Sage P and ZucmanRossi J: Genetic alterations associated with hepatocellular carcinomas define distinct pathways of hepatocarcinogenesis. Gastroenterology 120: 1763-1773, 2001.

39. Peifer M and Polakis P: Wnt signaling in oncogenesis and embryogenesis - a look outside the nucleus. Science 287: 16061609,2000

40. Polakis P: Wnt signaling and cancer. Genes Dev 14: 1837-1851, 2000.
41. Rubinfeld B, Souza B, Albert I, Müller O, Chamberlain SH, Masiarz FR, Munemitsu S and Polakis P: Association of the APC gene product with beta-catenin. Science 262: 1731-1734, 1993.

42. Korinek V, Barker N, Morin PJ, van Wichen D, de Weger R, Kinzler KW, Vogelstein B and Clevers H: Constitutive transcriptional activation by a beta-catenin-Tcf complex in APC $^{-/}$colon carcinoma. Science 275: 1784-1787, 1997.

43. Bienz $M$ and Clevers $\mathrm{H}$ : Linking colorectal cancer to Wnt signaling. Cell 103: 311-320, 2000

44. Miano JM, Long X and Fujiwara K: Serum response factor: master regulator of the actin cytoskeleton and contractile apparatus. Am J Physiol 292: C70-C81, 2007.

45. Chai J, Norng M, Tarnawski AS and Chow J: A critical role of serum response factor in myofibroblast differentiation during experimental oesophageal ulcer healing in rats. Gut 56: 621-630, 2007. 